\title{
The Correlation Between Technologies and Rating Scales in Gait Analysis
}

\author{
Ilaria Viscione, Francesca D’Elia, Rodolfo Vastola and Maurizio Sibilio \\ Department of Humanities, Philosophy and Education, University of Salerno, Fisciano, Salerno, Italy, 84084, Italy
}

\begin{abstract}
Gait is a key function of human movement which plays an important role in motion analysis. Both in the clinical field and in rehabilitation, gait analysis is useful to evaluate the parameters that are modified following the administration of a protocol of adapted physical activity (APA). Gait parameters could be measured using traditional rating scales, such as the Short Physical Performance Battery (SPPB) and technologies as a support to provide an assessment of gait quality. The aim of this study is to increase the objectivity of gait data obtained before and after a targeted APA program for a group of elderly people by integrating the traditional SPPB rating scale with the G Walk digital system. The former is an assessment tool to evaluate the functioning of lower extremity, in terms of chair stand, walk, and standing balance; whereas the latter can objectively evaluate the parameters of the gait. The sample was composed of 11 adults aged between 67 and 94 years. The participants were chosen on the basis of a number of tests carried out to analyze their levels of autonomy, intellectual capacities and motor functioning. It has planned a six months APA protocol: before and after it, SPPB and G-Walk were administered. The results showed that space-time characteristics generally improve after APA intervention; therefore, the use of technology is a useful support for the evaluation scales.
\end{abstract}

Key words: Gait analysis, short physical performance battery, G-Walk, adapted physical activity.

\section{Introduction}

Gait is a repetitive sequence of movement of the lower limbs in order to advance the body while maintaining the stability of the support. Since each sequence includes a series of interactions between the various segments of the lower limbs and the total body mass, these interactions can be studied from three different aspects: the first divides the gait cycle based on the contact of the foot to the ground; the second considers the spatio-temporal parameters, while the third examines the functional aspects [1].

As outlined by Whittle [2], studies on gait analysis have mostly concentrated on investigating difficulties in walking. A lot of studies, therefore, have aimed at using gait analysis to identify motor problems and to look for benefits of normal gait. With the improvement of methodologies for data collection, the major limitation hasn't been the high quality of data

Corresponding author: Ilaria Viscione, MA, Ph.D. candidate, research fields: methods and didactics of motor activities. production, but knowing how best to use this data for people's benefit. Therefore, both for clinical and scientific improvement, there has also been a growing interest in the use of observational or visual gait analysis [3].

Some examples of scales that have been developed to measure movement as part of a broader evaluation of disability include: Instrumental Activities of Daily Living (IADL) [4], the Barthel Index [5], Activities of Daily Living (ADL) [6], Tinetti Performance-Oriented Mobility Assessment (POMA) [7], and the Short Physical Performance Battery [8]. In addition to these scales, the new technologies are a useful support, which can complement and enrich the meaning of the extent of existing assessment tests. The integration of quantitative data comes from the need to use information that can be obtained using new technological systems [9] that provide data related to kinematics and dynamics which traditional scales do not supply, such as the orientation of the body segments, forces and displacements in space. 
Especially among the elderly, it is important to evaluate the mobility with standardized tools, such as structured assessment scales, and with the support of technological research tools to integrate traditional evaluations to provide more objective data. Moreover, the traditional rating scales used to analyze gait parameters are partly subjective since the researcher is required to observe the quality of a patient's gait by making him/her walk. In this case, the measure needs precise objective evaluation of different gait parameters that new technologies can give with efficient measurement. This is because they reduce the margin of error caused by subjective techniques [10]. The application of new technologies to the analysis of human movement, in fact, is an important support to traditional rating systems in order to provide an assessment of gait quality that can be used in combination with other measures to monitor the mobility, and as a predictive tool for possible disability [9].

\subsection{Objective}

The aim of this study is to integrate the traditional rating scales with digital systems with the correlation of gait data in older adults using two instruments: the Short Physical Performance Battery (SPPB) [8] and G Walk (BTS Bioengineering ${ }^{\circledR}$ ), before and after six months APA intervention. The former is an objective assessment tool for evaluating the functioning of lower limbs, in terms of standing balance, walk and chair stands, whereas the latter can objectively evaluate gait parameters. Therefore, referred to gait analysis, this pilot study aims to increase the reliability and validity of the data collected between the two instruments in order to support the observation with an instrumental analysis.

\section{Materials and Methods}

\subsection{Setting}

Project Freedom (Function of research on exergames to expand opportunities for educational mobility of the elderly) aimed at promoting physical activity and mobility in old age, to slow the decline in motor skills, which is evident also in gait characteristics. During project Freedom, promoted by the University of Salerno, a pilot study was carried out with the aim of correlating data collected on gait parameters of 11 adults between the ages of 67 and 94 years (Standard Deviation 14.8; Average 77.08). The participants came from an elderly people's home in Salerno, which provides social aid for the elderly [11].

\subsection{Participants}

Participants resided at the old people's home at the time. They were chosen on the basis of a number of tests carried out to analyze their levels of autonomy, intellectual capacities and motor functioning.

\subsection{Instruments}

Gait parameters were measured by the Short Physical Performance Battery (SPPB) and the G-Walk, before and after the implementation of the APA protocol.

The SPPB was administered by a specialist osteopath. This scale is a performance test using measures of walk (in a length of four meters), standing balance, and chair stands performance to assess lower extremity function: (1) To assess gait speed, participants were timed from a standing start and asked to walk at their normal speed over a 4-meter distance; (2) The standing balance is composed of three different balance tests, in increasing difficulty: feet together, semi-tandem stance and full-tandem stance. For each sub-test, it is required to maintain balance for 10 seconds, for a total of 30 seconds for the entire balance test; (3) The chair stand test can assess lower extremity strength; elders were asked to cross their arms in front of their chest and rise from a chair as quickly as they could, for five times. The score ranges from 0 (if the person is unable or takes longer than 60 seconds to complete the test) to a maximum score of 4 (if the test is carried out in less than 11.2 seconds) (Table 1). The 
Table 1 SPPB score.

\begin{tabular}{llll}
\hline & Walk & Standing balance & Chair stand \\
\hline 0 & unable & side-by-side stance & unable \\
1 & $>7.5$ & semi-tandem stance 0-9 & $>16.6$ \\
2 & $5.4-7.4$ & full-tandem stance 0-2 & $13.7-16.6$ \\
3 & $4.1-5.3$ & full-tandem stance 3-9 & $11.2-13.6$ \\
4 & $<4.1$ & full-tandem stance 10 & 11.2 \\
\hline
\end{tabular}

Table 2 Pre and Post Activity SPPB data.

\begin{tabular}{lll}
\hline Average & Pre-activity & Post-activity \\
\hline Walk & 3.4 & 3.9 \\
Balance & 3 & 2.9 \\
Chair Stand & 3.2 & 3.4 \\
Total & 9.5 & 10.25 \\
\hline
\end{tabular}

Table 3 Effects of the APA intervention in SPPB data.

\begin{tabular}{llll}
\hline & Increase & No variation & Decrease \\
\hline Balance & $25 \%$ & $38 \%$ & $38 \%$ \\
Gait & $31.25 \%$ & $68.75 \%$ & $0 \%$ \\
Sit to stand & $25 \%$ & $56.25 \%$ & $18.75 \%$ \\
Total & $37.50 \%$ & $43.75 \%$ & $18.75 \%$ \\
\hline
\end{tabular}

score of the overall performance is calculated by the sum of the three categories (range 0 to 12) [8].

The G-Walk is a digital gait analysis system which can consider the parameters of gait using a system consisting of an inertial sensor connected via Bluetooth to a computer that provides the possibility to define both the parameters of space and time of the rotation of the pelvis. It is an accelerometer to measure acceleration that can produce an output voltage or capacitance proportional to acceleration. The sensor is placed around the waist with an ergonomic belt. Hence, the subject is totally free to walk, run and jump [12].

\subsection{APA Protocol}

Adapted physical activity (APA) aims to prevent disease and to counter the damage caused by the physiological decline of psychomotor function in old age. Among the elderly, activity restriction is an independent predictor of decline in physical function. In recent years, in fact, the progressive aging of the population leads to reflections on the importance of the prevention of diseases related to aging, in order to promote active lifestyles among the elderly [11].
Engaging in adapted physical activity can also slow down the decline in mobility in old age, which is also evident in gait characteristics. In fact, a different locomotion can implicate incidences of fall [13-15]. In this study, APA intervention was structured twice a week in progressive more complex lessons that are possible to divide into three phases: neuromuscular activation (walking, sailing), nuclear phase (muscle strengthening exercises, joint mobility, coordination and balance) and cool down (walking, exercise inhaling and exhaling).

\section{Results and Analysis}

The results show that adaptive physical activity can have positive effects among the elderly in terms of: change in speed, cadence (number of steps per minute), single and double support duration, space-temporal characteristics, duration stance phase, change in phase of flight, and gradient acceleration. In particular, the data shows that space-time characteristics generally improve after the APA intervention. This has been evidently recorded both in the SPPB data as well as in G-Walk (Table 2).

SPPB data generally improved after APA intervention (Table 3). The sum of the average of data obtained of the three subtests show higher scores regarding the functionality of the lower limbs.

Referred to the output results, it is possible to extract percentages clarifying the effects of the protocol APA carried out.

The correlation between SPPB and G-Walk is evident when we compare the positive results of gait speed and cadence with the improvements in gait SPPB test. With reference to the "no variation" data, the stability of the motor performance in the elderly is a positive indicator because a simple slowing or stopping the physiological decline of motor functions, is still a positive result among the elderly [16].

In relation to the speed of walking, it is possible to standardize the scores on a scale ranging from 0 to 4 , also for G-Walk data. 
Starting from the path of SPPB subtest, it's possible to derive the data speed using the formula speed $=$ $\frac{\text { Space (4 meters) }}{\text { Time }}$ (Table 4).

As with the data relating to SPPB speed scores, also the scores obtained through G-Walk can be assigned a score ranging from 0 to 4 for the motor performance so as to make the correlation between the two possible. Table 5 provides the ranges.

Table 4 Relation between time and speed.

\begin{tabular}{llll}
\hline Time & 7.5 & 5.4 & 4.1 \\
\hline Speed & 0.533333 & 0.740741 & 0.97561 \\
\hline
\end{tabular}

Table 5 Assignment of points based on speed.

\begin{tabular}{lllllll}
\hline Speed & unable & $\mathrm{S}<0.533$ & $\begin{array}{l}0.533< \\
\mathrm{S}<0.74\end{array}$ & $\begin{array}{l}0.74<0.97 \\
\text { Ranges }\end{array}$ & & $\mathrm{S}>0.97$ \\
\hline $\begin{array}{l}\text { Score } \\
\text { Attributed }\end{array}$ & 0 & 1 & 2 & 3 & 4 \\
\hline
\end{tabular}

Table 6 SPPB and G-Walk results.

\begin{tabular}{|c|c|c|c|c|}
\hline \multirow[b]{2}{*}{ Sample } & \multicolumn{2}{|c|}{$\begin{array}{c}\text { G-Walk speed } \\
\text { Scores - Raw Data }\end{array}$} & \multicolumn{2}{|c|}{$\begin{array}{c}\text { G-Walk speed scores } \\
\text { using SPPB Scale Ranges }\end{array}$} \\
\hline & In & Out & In & Out \\
\hline $\mathrm{a}$ & 0.77 & 1.08 & 3 & 4 \\
\hline b & 0.48 & 0.98 & 0 & 4 \\
\hline C & 1.16 & 1.44 & 4 & 4 \\
\hline d & 0.95 & 1.13 & 3 & 4 \\
\hline e & 1.01 & 1.24 & 4 & 4 \\
\hline $\mathrm{f}$ & 0.73 & 1.02 & 2 & 4 \\
\hline g & 0.8 & 0.85 & 3 & 3 \\
\hline h & 0.56 & 1.05 & 2 & 4 \\
\hline $\mathrm{i}$ & 0.51 & 0.91 & 0 & 3 \\
\hline $\mathrm{j}$ & 0.63 & 1 & 2 & 4 \\
\hline $\mathrm{k}$ & 0.62 & 0.63 & 2 & 2 \\
\hline
\end{tabular}

Table 7 SPPB and G-Walk correlation data.

\begin{tabular}{lllll}
\hline \multirow{2}{*}{ Sample } & \multicolumn{2}{c}{ pre-activity data (In) } & \multicolumn{2}{c}{ post-activity data (Out) } \\
\cline { 2 - 5 } & SPPB & G-Walk & SPPB & G-Walk \\
\hline a & 3 & 3 & 4 & 4 \\
b & 4 & 0 & 4 & 4 \\
c & 4 & 4 & 4 & 4 \\
d & 4 & 3 & 4 & 4 \\
e & 4 & 4 & 4 & 4 \\
f & 2 & 2 & 4 & 4 \\
g & 3 & 3 & 4 & 3 \\
h & 2 & 2 & 2 & 4 \\
i & 4 & 0 & 4 & 3 \\
j & 4 & 2 & 4 & 4 \\
k & 4 & 2 & 4 & 2 \\
\hline
\end{tabular}

The ranges in Table 5 were used to produce the following data (Table 6), converting G-Walk raw scores into speed scores using the SPPB Scale ranges.

The correlation of the data obtained regarding speed, using both SPPB and G-Walk at pre-activity (in) and post-activity (out) phases is presented below (Table 7).

In $54 \%$ of the cases, there is an exact match of the pre-activity data between the instruments used; the correspondence of the post-activity data is of $64 \%$.

\section{Discussion and Conclusions}

The link between inactivity and health can lead to hypokinetic diseases. Thus, an increase in physical activity can improve the quality of life among the elderly. In fact, there are a lot of reasons to practice structured and regular physical activity among elders, to improve conditions of diseases such as obesity, diabetes, hypertension, cardio-vascular disease, osteoporosis and so on [17].

A structured adaptive physical activity also produces improvements in balance and gait $[18,19]$. A lot of gait characteristics change with aging. With age, for example, a decline occurs in gait speed, particularly in step or stride length rather than a decrease in cadence. Also after age 75 or 80 , other characteristics are evident such as shorter and broader strides, decreased vertical and increased horizontal head excursions, decreased limb excursions, longer stance and shorter swing durations and slower cadence [20].

Although this research can be considered a pilot study, it is important to highlight the validity of an objective evaluation in gait analysis for the elderly, especially before and after the application of a structured protocol of adapted physical activity. The parameters taken into consideration for this research demonstrate the possibility of quantifying possible variations in elderly gait.

It would be interesting to continue research to compare the parameters obtained as a result of observation and administration of rating scales with the traditional support of the data provided by the new 
technologies. The study opens interesting prospects for the future because it shows that the instrumental analysis is a useful support for rating scales.

\section{References}

[1] Perry, J. 1992. Gait Analysis. Normal and Pathological Function (Second edition). New York: Slack Inc.

[2] Whittle, M. W. 2014. Gait Analysis: An Introduction. Oxford: Butterworth-Heinemann.

[3] Benedetti, M. G. 2002. "An Update on Clinical Gait Analysis.” European Journal of Physical and Rehabilitation Medicine 38 (3): 117.

[4] Lawton, M. P., and Brody, E. M. “Assessment of Older People: Self-maintaining and Instrumental Activities of Daily Living.” The Gerontologist 9 (3): 1969.

[5] Mahoney, F. I., and Barthel, D. W. 1965 "Functional Evaluation: The Barthel Index.” Md. State Med. J. 14: 56-61.

[6] Katz, S., Ford, A. B., Moskowitz, R. W., Jackson, B. A., and Jaffe, M. W. 1963 "Studies of Illness in the Aged: The Index of ADL: A Standardized Measure of Biological and Psychosocial Function.” JAMA 185 (12): 914-9.

[7] Tinetti, M. E. 1986. "Performance-oriented Assessment of Mobility Problems in Elderly Patients.” J. Am. Geriatr. Soc. 34 (2): 119-26.

[8] Guralnik, J. M., Simonsick, E. M., Ferrucci, L., Glynn, R. J., Berkman, L. F., Blazer, D. G., Scherr, P.A., and Wallace, R. B. 1994. "A Short Physical Performance Battery Assessing Lower Extremity Function: Association with Self-reported Disability and Prediction of Mortality and Nursing Home Admission.” J. Gerontol. 49 (2): M85-M94.

[9] Vastola, R. 2014. The Potential of the Technology of Motion Analysis in Assessing Teaching. Lecce: Pensa Editore. (in Italy)

[10] Muro-de-la-Herran, A., Garcia-Zapirain, B., and Mendez-Zorrilla, A. 2014. "Gait Analysis Methods: An Overview of Wearable and Non-wearable Systems, Highlighting Clinical Applications.” Sensors 14 (2): 3362-94.
[11] D’Elia, F., Vastola, R., and Sibilio, M. 2014. “The Use of Exergames for Adaptive Physical Activity among the Elderly.” Journal of Sports Science 2 (1): 47-52.

[12] Bondre, N., Cerutti A., and Major, M. 2014. Accelerometer Systems Northwestern University Prosthetics-Orthotics Center (NUPOC) Poster Presentation.

[13] Van Het Reve, E., and De Bruin, E. D. 2014. "Strength-balance Supplemented with Computerized Cognitive Training to Improve Dual Task Gait and Divided Attention in Older Adults: A Multicenter Randomized-Controlled Trial.” BMC Geriatrics 14 (1): 134.

[14] Fernandes, B., Ferreira, M. J., Batista, F., Evangelista, I., Prates, L., and Silveira-Sérgio, J. 2015. “Task-oriented Training and Lower Limb Strengthening to Improve Balance and Function after Stroke: A Pilot Study.” The European Journal of Physiotherapy 17 (2): 74-80.

[15] Fang, W. C., Sheu, F. R., Lin, Y. L., Lee, Y. L., and Chen, N. S. 2015. "Interactive Physical Games: Improving Balance in Older Adults.” In Smart Learning Environments, Springer Berlin Heidelberg, 159-74.

[16] Buchman A. S., Boyle P. A., Wilson R. S., Bienias J. L., and Bennett, D. A. 2007. "Physical Activity and Motor Decline in Older Persons.” Muscle \& Nerve 35 (3): 354-62.

[17] Steadward, R. D., Watkinson, E. J., and Wheeler, G. D. 2003. Adapted Physical Activity. Aberta: University of Alberta Press.

[18] Michael, K., Goldberg, A. P., Treuth, M. S., Beans, J., Normandt, P., and Macko, R. F. 2009. "Progressive Adaptive Physical Activity in Stroke Improves Balance, Gait, and Fitness: Preliminary Results.” Topics in Stroke Rehabilitation 16 (2): 133-9.

[19] Pau, M., Leban, B., Collu, G., and Migliaccio, G. M. 2014 "Effect of Light and Vigorous Physical Activity on Balance and Gait of Older Adults.” Arch. Gerontol. Geriatr. 59 (3): 568-73.

[20] Alexander, N. B. 1996. "Gait Disorders in Older Adults." Journal of the American Geriatrics Society 44 (4): 434-51. 